\title{
Impact of Vlasov Foundation Parameters on the Deflection of a Non-uniform Timoshenko Beam Subject to a Moving Load
}

\author{
Magdalena Ataman ${ }^{1 *}$ \\ ${ }^{1}$ Institute of Roads and Bridges, \\ Faculty of Civil Engineering, Warsaw University of Technology, \\ 16 Armii Ludowej Ave., 00-637 Warsaw, Poland \\ * Corresponding author, e-mail: m.ataman@il.pw.edu.pl
}

Received: 09 October 2018, Accepted: 02 April 2019, Published online: 28 May 2019

\begin{abstract}
Subject of the study is a Timoshenko beam with transverse-variable Young's modulus. Problem of vibrations of the beam resting on an inertial Vlasov foundation and subjected to a moving force is solved analytically. The Timoshenko beam's eigenproblem is discussed and the physical sense of the additional band of natural vibrations and the corresponding critical frequency is analytically explained. The impact of the foundation and its parameters on the Timoshenko beam's vibrations forced by moving force is investigated. Dynamic factors relating to beam deflections are analyzed. Two vibration cases are discussed: forced vibrations (when a moving force is applied to the beam) and free vibrations (after the moving force has been left the beam). The damping effect on vibration is taken into account in the problem solution. The results indicate that appropriate selection of the foundation's parameters allows for the beam deflection's significant reduction, while the impact of the shear coefficient in the foundation on the reduction is more pronounced than the impact of other factors.
\end{abstract}

Keywords

non-uniform Timoshenko beam, inertial Vlasov foundation, moving force

\section{Introduction}

In 1921-1922, basing on Rayleigh's assumptions, Timoshenko published two papers $[1,2]$, on the influence of a beam section shear and rotary inertia effect on its transverse vibrations. These studies had been known much earlier in Russia, published in 1914-1916, and after Timoshenko's death republished by Grigoljuk in the 1970s in book series.

Timoshenko's publications played a very important role in the development of dynamics of structures in the twentieth century [3]. In the early 1950s, three decades after the publication of Timoshenko papers, Mindlin published several important studies devoted to the Timoshenko model's application to the dynamics of thick plates. All these publications have been summarized in Mindlin's monograph [4].

To date, several thousand studies have been published directly or indirectly concerning beams and thick plates using the Timoshenko model's idea. Many authors have addressed the problem of the Timoshenko beam natural vibration's second and third bands, e.g. [3, 5-8]. Also, moving loads on the Timoshenko beam were analyzed in numerous papers, including [9-16]. Some of the relevant literature refers to the Timoshenko beam resting on various types of deformable foundation, e.g. [11, 13-16]. However, in most studies available in the literature, the foundation inertia is neglected.

This paper discusses the Timoshenko beam's eigenproblem and the analytically explained physical sense of the additional band of natural vibrations and the corresponding so-called critical frequency $\omega_{c r}$. The effect of the adopted deformable foundation model and its characteristics (elasticity, shear, inertia) on the Timoshenko beam's deflections under a moving load is investigated. Subject of the study is a beam with transverse-variable Young's modulus.

\section{Governing equations}

The Timoshenko beam motion equation can be derived in many ways. Locally, by the kinetostatic method, with attached forces and moments of inertia, using the virtual work principle, from the Lagrange equations of the second 
kind or using the Hamilton principle. The motion equations can also be derived by imposing some kinematic hypotheses on displacements, as, for example, in a study [5], and other studies.

The constraints equation and relations between internal forces and displacements in the Timoshenko beam are given by the Eqs. (1-3):

$\frac{\partial w(x, t)}{\partial x}=\psi(x, t)+\beta(x, t)$,

$M_{x}(x, t)=-E J \frac{\partial \psi(x, t)}{\partial x}$,

$T_{x}(x, t)=\kappa G A \beta(x, t)$,

where $\psi$ and $\beta$ are the angles of the beam cross-section rotation due to pure bending and shearing, respectively. Whereas $w$ is the deflection of a beam with Young's modulus equal to $E$, cross-sectional area $A$ and moment of inertia $J, G$ is the Kirchhoff modulus, and $\kappa$ is the shear coefficient.

Listing and comparison of correction coefficients $\kappa$ in the Timoshenko beam, used by various authors, are presented by Bystrzycki in [17]. Some authors, following Mindlin [4] and Mindlin and Deresiewicz [18], instead of the shear factor, have introduced the effective shear modulus to the Timoshenko beam equations.

Two Timoshenko beam motion equations, without damping, under load $p=p(x, t)$ can be formulated as:

$E J \frac{\partial^{2} \psi}{\partial x^{2}}+\kappa G A\left(\frac{\partial w}{\partial x}-\psi\right)=\rho J \frac{\partial^{2} \psi}{\partial t^{2}}$,

$\kappa G A\left(\frac{\partial^{2} w}{\partial x^{2}}-\frac{\partial \psi}{\partial x}\right)+p=\rho A \frac{\partial^{2} w}{\partial t^{2}}$.

The equations of motion Eqs. (4) and (5) can be transformed to reduce them to a single double-wave equation describing either the beam's total deflection :

$$
\begin{aligned}
& E J \frac{\partial^{4} w}{\partial x^{4}}+\rho A \frac{\partial^{2} w}{\partial t^{2}}-\rho J\left(1+\frac{E}{\kappa G}\right) \frac{\partial^{4} w}{\partial x^{2} \partial t^{2}}+\frac{\rho^{2} J}{\kappa G} \frac{\partial^{4} w}{\partial t^{4}} \\
& =p-\frac{E J}{\kappa G A} \frac{\partial^{2} p}{\partial x^{2}}+\frac{\rho J}{\kappa G A} \frac{\partial^{2} p}{\partial t^{2}}
\end{aligned}
$$

or its cross-section rotation angle $\psi$ :

$$
E J \frac{\partial^{4} \psi}{\partial x^{4}}+\rho A \frac{\partial^{2} \psi}{\partial t^{2}}-\rho J\left(1+\frac{E}{\kappa G}\right) \frac{\partial^{4} \psi}{\partial x^{2} \partial t^{2}}+\frac{\rho^{2} J}{\kappa G} \frac{\partial^{4} \psi}{\partial t^{4}}=\frac{\partial p}{\partial x} .
$$

In this study, Eq. (6) with an unknown deflection $w$ is analyzed.
Eqs. (6) and (7) can be rewritten by entering two wave velocities $c_{1}{ }^{2}=E / p$ and $c_{2}{ }^{2}=\kappa G / p$, as well as inertia radius $\tilde{r}^{2}=J / A$, as in [5].

Four boundary conditions and four initial conditions must be attached to the motion equations so that the principle of beam motion specificity is retained.

\section{Eigenproblem of Timoshenko beam}

To solve the Timoshenko beam eigenproblem, spatial and temporal variables are separated in homogeneous Eqs. (4) and (5): $w(x, t)=W(x) T(t), \psi(x, t)=\Psi(x) T(t)$, which produces system of equations:

$E J \Psi^{\prime \prime} T+\kappa G A\left(W^{\prime}-\Psi\right) T-\rho J \Psi \ddot{T}=0$,

$\kappa G A\left(W^{\prime \prime}-\Psi^{\prime}\right) T-\rho A W \ddot{T}=0$.

Assuming harmonic motion of natural vibrations, after substitution of $\ddot{T}=-\omega^{2} T$ into Eqs. (8) and (9), the following equations are obtained $[7,8]$ :

$E J \Psi^{\prime \prime}+\kappa G A\left(W^{\prime}-\Psi\right)+\omega^{2} \rho J \Psi=0$,

$\kappa G A\left(W^{\prime \prime}-\Psi^{\prime}\right)+\rho A \omega^{2} W=0$.

Equations (10) and (11) can be transformed and reduced to two fourth order equations with function $W$ or $\Psi$ :

$$
\begin{aligned}
& E J W^{I V}+\rho \omega^{2} J(E / \kappa G+1) W^{\prime \prime} \\
& +\rho \omega^{2} A\left(\omega^{2} \rho \tilde{r}^{2} / \kappa G-1\right) W=0, \\
& E J \Psi^{I V}+\rho \omega^{2} J(E / \kappa G+1) \Psi " \\
& +\rho \omega^{2} A\left(\omega^{2} \rho \tilde{r}^{2} / \kappa G-1\right) \Psi=0 .
\end{aligned}
$$

It is easy to see that Eqs. (12) and (13) are identical. Also, identical must be their characteristic equations defining wave numbers of $\mathrm{m}^{-1}$ dimension and natural circular frequencies.

By solving characteristic equation of Eq. (12) or (13), four roots are determined:

$$
\begin{aligned}
& s_{1,2,3,4}= \pm\left\{-\frac{\rho \omega^{2}}{2}\left(\frac{1}{\kappa G}+\frac{1}{E}\right)\right. \\
& \left. \pm \sqrt{\left[\frac{\rho \omega^{2}}{2}\left(\frac{1}{\kappa G}+\frac{1}{E}\right)\right]^{2}+\frac{\rho \omega^{2}}{E}\left(\frac{1}{\tilde{r}^{2}}-\frac{\rho \omega^{2}}{\kappa G}\right)}\right\}^{\frac{1}{2}} .
\end{aligned}
$$

Two of them $s_{1}$ and $s_{2}$ will always be imaginary and the other two $s_{3}$ and $s_{4}$ may be real, imaginary or equal to zero, depending on the beam's material constants and geometric characteristics. 
The boundary between the real and imaginary roots is the last component in Eq. (14). Assuming that in a specific case the expression in brackets can be equal to zero, the Timoshenko beam's so-called critical natural frequency $\omega_{c r}$ can be determined, $s_{3,4}=0$ hence:

$\omega^{2}=\omega_{c r}^{2}=\frac{\kappa G}{\rho \tilde{r}^{2}}=\frac{\kappa G A}{\rho J}=\left(\frac{c_{2}}{\tilde{r}}\right)^{2}$.

If $\omega<\sqrt{\kappa G A / \rho J}$, i.e. $\omega<\omega_{c r}$, the characteristic equation roots $s_{3}$ and $s_{4}$ will be real. If, in turn, $\omega>\sqrt{\kappa G A / \rho J}$, i.e. $\omega>\omega_{c r}$, then roots and will be imaginary. The case of $\omega=\omega_{c r}$ refers to the third band of Timoshenko beam's natural vibration. In these three cases, the natural vibration will, of course, assume different modes. Timoshenko beam's natural vibrations with first and second frequency are discussed in detail in [7].

In the case of the third band of Timoshenko beam's natural vibration $\omega=\omega_{c r}=c_{2} / \tilde{r}$, Eqs. (12) and (13) are:

$$
\begin{aligned}
& E J W^{I V}+A(E+\kappa G) W^{\prime \prime}=0, \\
& E J \Psi^{I V}+A(E+\kappa G) \Psi^{\prime \prime}=0 .
\end{aligned}
$$

The characteristic equations of Eqs. (16) and (17) are the same, and their solutions have the form

$s_{1,2}=0$,

$S_{3,4}= \pm \sqrt{-A(E+\kappa G) / E J}$.

The solution of Eq. (16) is the following function

$W=A_{1}+A_{2} x+A_{3} \sin \theta x+A_{4} \cos \theta x$,

where $\theta=\sqrt{A(E+\kappa G) / E J}$ is a wave number.

The solution of Eq. (17) it is identical, except for constants.

\section{Vibrations of Timoshenko beam on Vlasov foundation due to moving load}

Two basic approaches to solving the problem of Timoshenko beam's free and forced vibration are reported in the literature. The first way, applied by Mindlin and his School, is to analyze the system of two differential motion equations (Eqs. (4) and (5)), in which time derivatives are of the second order, along with four initial conditions for the looked-for functions and their derivatives, according to the motion specificity principle. Whereas in Russian studies the equations system (Eqs. (4) and (5)) is transformed into one Eq. (6), Eq. (7) or into an equation in the wave form. These are equations with elevated fourth order of the time derivative.

\subsection{Equation of motion of non-uniform beam}

In this paper, the Eq. (6) of Timoshenko beam on a deformable foundation is analyzed and solved. Layer of an inertial foundation with thickness $H$ is described by factors $k_{s}, G_{s}$ and $m_{s}$. Factor $k_{s}$ describes the foundation's elastic settlement, factor $G_{s}$ determines the effect of shear in the foundation and is therefore a measure of the load's transfer to the foundation in the vicinity of its application, and $m_{s}$ represents the foundation's inertia. A foundation so characterized can be described by Vlasov's inertial model [19]. Foundation's dynamic reaction to beam $r(x, t)$ and load $q(x, t)$ are formulated as:

$$
\begin{aligned}
& r(x, t)=k_{s} w(x, t)-G_{s} \frac{\partial^{2} w(x, t)}{\partial x^{2}}+m_{s} \frac{\partial^{2} w(x, t)}{\partial t^{2}}, \\
& q(x, t)=P \delta(x, v t), \\
& p(x, t)=q(x, t)-r(x, t), \\
& p(x, t)=P \delta(x-v t) \\
& -\left[k_{s} w(x, t)-G_{s} \frac{\partial^{2} w(x, t)}{\partial x^{2}}+m_{s} \frac{\partial^{2} w(x, t)}{\partial t^{2}}\right] .
\end{aligned}
$$

For a beam with Young's modulus variable after function $E(z)$ or stepwise, in the equations describing its vibration, $E J$ should be replaced with equivalent stiffness

$\overline{E J}=\int_{-h / 2-e}^{h / 2-e} b(z) z^{2} E(z) d z$,

where:

$$
e=\int_{-h / 2}^{h / 2} E(z) z d z / \int_{-h / 2}^{h / 2} E(z) d z
$$

or

$e=\sum_{i=1}^{n}\left(E_{i} \int_{-h / 2+H_{i-1}}^{-h / 2+H_{i}} z d z\right) / \sum_{i=1}^{n}\left(E_{i} \int_{-h / 2+H_{i-1}}^{-h / 2+H_{i}} d z\right)$,

$H_{i}=\sum_{j=1}^{i} h_{j}$

Vertical displacements $w$ then refer to the beam's neutral axis, which is set off by $e$ relative to the uniform beam's neutral axis [20].

Upon consideration in Eq. (6) of the foundation's response and the beam's load in the form of a force moving at a constant velocity Eqs. (21)-(24), the equation of the non-uniform Timoshenko beam's vibration assumes the form of 


$$
\begin{aligned}
& \overline{E J}\left(1+\frac{G_{s}}{\kappa G A}\right) \frac{\partial^{4} w}{\partial x^{4}}-\left[\rho\left(J+\frac{\overline{E J}}{\kappa G}+\frac{G_{s} J}{\kappa G A}\right)\right. \\
& \left.+m_{s} \frac{\overline{E J}}{\kappa G A}\right] \frac{\partial^{4} w}{\partial x^{2} \partial t^{2}}+\frac{\rho J}{\kappa G}\left(\rho+\frac{m_{s}}{A}\right) \frac{\partial^{4} w}{\partial t^{4}} \\
& -\left(\frac{\overline{E J} k_{s}}{\kappa G A}+G_{s}\right) \frac{\partial^{2} w}{\partial x^{2}}+\left(\rho A+\frac{k_{s} \rho J}{\kappa G A}+m_{s}\right) \frac{\partial^{2} w}{\partial t^{2}}+k_{s} w \\
& =\left(1-\frac{\overline{E J}}{\kappa G A} \frac{\partial^{2}}{\partial x^{2}}+\frac{\rho J}{\kappa G A} \frac{\partial^{2}}{\partial t^{2}}\right) P \delta(x-v t) .
\end{aligned}
$$

The motion equation, given by Eq. (29), is a partial differential equation of the fourth order with regard to its spatial and temporal co-ordinates.

\subsection{Solution of beam's forced vibration problem}

In the case of boundary conditions of simple support of a beam with length $l$ and assuming that the beam vibrations are harmonic, the analytic solution of Eq. (29) can be formulated as

$$
\begin{aligned}
& w(x, t)=\sum_{n=1}^{\infty}\left(C_{1 n} \cos \omega_{1 n} t+C_{2 n} \sin \omega_{1 n} t\right. \\
& \left.+C_{3 n} \cos \omega_{2 n} t+C_{4 n} \sin \omega_{2 n} t+K_{1 n} \sin \frac{n \pi v t}{l}\right) \sin \frac{n \pi x}{l},
\end{aligned}
$$

where $\omega_{1 n}$ and $\omega_{2 n}$ are the beam's natural frequencies:

$$
\begin{aligned}
& \omega_{1 n}=\left[\frac{1}{2}\left(A_{1 n}-\sqrt{A_{1 n}^{2}-4 A_{2 n}}\right)\right]^{1 / 2}, \\
& \omega_{2 n}=\left[\frac{1}{2}\left(A_{1 n}+\sqrt{A_{1 n}^{2}-4 A_{2 n}}\right)\right]^{1 / 2},
\end{aligned}
$$

and

$$
\begin{aligned}
& K_{1 n}=2 P\left(S_{1 n}+S_{2 n}\right) / l\left(S_{3 n}-S_{4 n}+S_{5 n}\right), \\
& S_{1 n}=\kappa G A, \\
& S_{2 n}=\alpha_{n}^{2}\left(\overline{E J}-\rho v^{2} J\right), \\
& S_{3 n}=\overline{E J} \alpha_{n}^{2}\left(k_{s}+G_{s} \alpha_{n}^{2}\right) \\
& +\kappa G A\left(k_{s}+G_{s} \alpha_{n}^{2}+\overline{E J} \alpha_{n}^{4}\right), \\
& S_{4 n}=v^{2} \alpha_{n}^{2}\left\{\kappa G A\left(m_{s}+\rho A\right)+\rho J k_{s}\right. \\
& \left.+\left[\overline{E J}\left(m_{s}+\rho A\right)+\rho G_{s} J+\rho \kappa G A J\right] \alpha_{n}^{2}\right\}, \\
& S_{5 n}=v^{4} \alpha_{n}^{4} J \rho\left(m_{s}+\rho A\right),
\end{aligned}
$$

$$
\begin{aligned}
& A_{1 n}=\frac{1}{\rho J}\left[\left(\overline{E J}+\rho J \frac{G_{s}+\kappa G A}{m_{s}+\rho A}\right) \alpha_{n}^{2}\right. \\
& \left.+\kappa G A+\frac{\rho k_{s} J}{m_{s}+\rho A}\right], \\
& A_{2 n}=\frac{1}{\rho J\left(m_{s}+\rho A\right)}\left[\overline{E J}\left(\kappa G A+G_{s}\right) \alpha_{n}^{4}\right. \\
& \left.+\left(\overline{E J} k_{s}+\kappa G A G_{s}\right) \alpha_{n}^{2}+\kappa G A k_{s}\right], \\
& \alpha_{n}=\frac{n \pi}{l} .
\end{aligned}
$$

In a specific case of Eqs. (31) and (32), with $A_{1 n}^{2}=4 A_{2 n}$, there is $\omega_{1 n}=\omega_{2 n}=\omega_{l}=\sqrt{A_{1 n} / 2}$. The natural vibration mode corresponding to this frequency is called the third, additional band of Timoshenko beam vibrations [6, 7].

Constants $C_{1 n}, C_{2 n}, C_{3 n}$, and $C_{4 n}$ in Eq. (30) should be determined from the initial conditions of the problem, which after load decomposition $P \delta(x-v t)$ into sine Fourier series can be formulated as

$\left.w(x-t)\right|_{t=0}=0$,

$\left.\frac{\partial w(x, t)}{\partial t}\right|_{t=0}=0$,

$\left.\frac{\partial^{2} w(x, t)}{\partial t^{2}}\right|_{t=0}=0$

$\left.\frac{\partial^{3} w(x, t)}{\partial t^{3}}\right|_{t=0}=\frac{2 P \alpha_{n} v}{\rho A l}$.

Consideration of Eqs. (42)-(45) gives the solution of Eq. (29).

$$
\begin{aligned}
& w(x, t) \\
& =\sum_{n=1}^{\infty}\left[-v \alpha_{n} \frac{2 P+K_{1 n} A l \rho\left(v^{2} \alpha_{n}^{2}-\omega_{2 n}^{2}\right)}{A l \rho \omega_{1 n}\left(\omega_{1 n}^{2}-\omega_{2 n}^{2}\right)} \sin \omega_{1 n} t\right. \\
& +v \alpha_{n} \frac{2 P+K_{1 n} A l \rho\left(v^{2} \alpha_{n}^{2}-\omega_{1 n}^{2}\right)}{A l \rho \omega_{2 n}\left(\omega_{1 n}^{2}-\omega_{2, n}^{2}\right)} \sin \omega_{2 n} t \\
& \left.+K_{1 n} \sin \alpha_{n} v t\right] \sin \alpha_{n} x,
\end{aligned}
$$

where $K_{1 n}$ is given by Eq. (33).

With the assumption that all four initial conditions equal to zero, the beam deflection expression slightly differs. This has been assumed, inter alia, by Mackertich [12]. The initial conditions for Timoshenko beam and their 
impact on the forced vibration solution are discussed by Szcześniak [21], who has proven that both approaches lead practically to the same results.

Solution given by Eq. (46) is valid if a force moving at a velocity lower than the critical one is applied to the beam. After the load descending from the beam its free vibrations can be described as

$$
\begin{aligned}
& \tilde{w}(x, t)=\sum_{n=1}^{\infty}\left(\tilde{C}_{1 n} \cos \omega_{1 n} t+\tilde{C}_{2 n} \sin \omega_{1 n} t\right. \\
& \left.+\tilde{C}_{3 n} \cos \omega_{2 n} t+\tilde{C}_{4 n} \sin \omega_{2 n} t\right) \sin \frac{n \pi x}{l} .
\end{aligned}
$$

Integration constants $\tilde{C}_{1 n}-\tilde{C}_{4 n}$ are determined from the deflection continuity conditions and its three subsequent derivatives at time $t=l / v$, i.e. when the moving force leaves the beam. To determine the beam midpoint's deflection $(x=l / 2)$, the following conditions must be met:

$$
\begin{aligned}
& \left.w\left(\frac{l}{2}, t\right)\right|_{t=l / v}=\left.\tilde{w}\left(\frac{l}{2}, t\right)\right|_{t=l / v}, \\
& \left.\frac{d}{d t} w\left(\frac{l}{2}, t\right)\right|_{t=l / v}=\left.\frac{d}{d t} \tilde{w}\left(\frac{l}{2}, t\right)\right|_{t=l / v}, \\
& \left.\frac{d^{2}}{d t^{2}} w\left(\frac{l}{2}, t\right)\right|_{t=l / v}=\left.\frac{d^{2}}{d t^{2}} \tilde{w}\left(\frac{l}{2}, t\right)\right|_{t=l / v}, \\
& \left.\frac{d^{3}}{d t^{3}} w\left(\frac{l}{2}, t\right)\right|_{t=l / v}=\left.\frac{d^{3}}{d t^{3}} \tilde{w}\left(\frac{l}{2}, t\right)\right|_{t=l / v} .
\end{aligned}
$$

Solving the system of four Eqs. (48)-(51) produces the sought coefficients $\tilde{C}_{1 n}-\tilde{C}_{4 n}$.

As seen from solution Eq. (46), the beam deflection consists of free vibration with circular frequencies $\omega_{1 n}$ and $\omega_{2 n}$, and purely forced vibrations with circular frequency $\alpha_{n} v$. Components containing frequency $\omega_{2 n}$ are of relatively small values compared to other components, and in practical applications are sometimes neglected [1]. Graphical comparison of deflections resulting from beam vibrations with frequencies $\omega_{1 n}, \omega_{2 n}$ and $\alpha_{n} v$ is presented in Fig. 1. The geometric and material data were adopted as in [12]. Moving force velocity equals $v=50 \mathrm{~m} / \mathrm{s}$.

The charts in Fig. 1 clearly show that the deflections from the vibration at circular frequency $\omega_{2 n}$ are small compared to the forced vibration as well as the associated free vibration at frequency $\omega_{1 n^{n}}$. The maximum amplitude of vibrations at frequency $\omega_{2 n}$ amounts to $10^{-6}$ of the forced vibration amplitude, and the amplitude of free

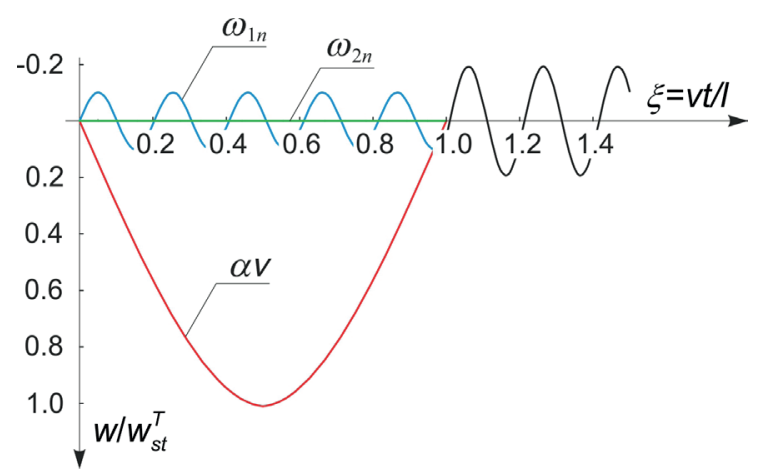

Fig. 1 Timoshenko beam midpoint deflection. Vibration decomposition into purely forced vibration at a circular frequency $\alpha_{n} v$ and associated free vibrations at circular frequencies $\omega_{1 n}$ and $\omega_{2 n}$

vibrations at frequency $\omega_{1 n}$ is five orders smaller. Thus, neglecting components containing $\omega_{2 n}$ in practical applications it is justified.

\subsection{Damped vibrations}

The above analysis concerns Timoshenko beam's undamped vibrations. Free vibration equations for a Timoshenko beam made of Voigt or Maxwell visco-elastic material, or the material described by the three-parameter standard model were given and analyzed, inter alia, by Szcześniak [5] under simplifying assumptions $\tau=\eta / E=\eta_{T} / G$. The equation for a beam made of Voigt material resting on Vlasov foundation and subjected to a moving force can, on the basis of [5], formulated as

$$
\begin{aligned}
& \overline{E J}\left[\left(1+\tau \frac{\partial}{\partial t}\right)^{2}+\frac{G_{s}}{\kappa G A}\left(1+\tau \frac{\partial}{\partial t}\right)\right] \frac{\partial^{4} w}{\partial x^{4}} \\
& -\rho\left[\left(J+\frac{\overline{E J}}{\kappa G}\right)\left(1+\tau \frac{\partial}{\partial t}\right)+\frac{G_{s} J}{\kappa G A}\right. \\
& \left.+m_{s} \frac{\overline{E J}}{\kappa G A}\left(1+\tau \frac{\partial}{\partial t}\right)\right] \frac{\partial^{4} w}{\partial x^{2} \partial t^{2}}+\frac{\rho J}{\kappa G}\left(\rho+\frac{m_{s}}{A}\right) \frac{\partial^{4} w}{\partial t^{4}} \\
& +\frac{\rho J c}{\kappa G A} \frac{\partial^{3} w}{\partial t^{3}}-\frac{\overline{E J} c}{\kappa G A}\left(1+\tau \frac{\partial}{\partial t}\right) \frac{\partial^{3} w}{\partial x^{2} \partial t} \\
& -\left(1+\tau \frac{\partial}{\partial t}\right)\left(G_{s}+\frac{\overline{E J} k_{s}}{\kappa G A}\right) \frac{\partial^{2} w}{\partial x^{2}} \\
& +\left[\rho A\left(1+\tau \frac{\partial}{\partial t}\right)+\frac{\rho J k_{s}}{\kappa G A}+m_{s}\right] \frac{\partial^{2} w}{\partial t^{2}} \\
& +c\left(1+\tau \frac{\partial}{\partial t}\right) \frac{\partial w}{\partial t}+k_{s}\left(1+\tau \frac{\partial}{\partial t}\right) w=\left[\left(1+\tau \frac{\partial}{\partial t}\right)\right. \\
& \left.-\frac{\overline{E J}}{\kappa G A}\left(1+\tau \frac{\partial}{\partial t}\right) \frac{\partial^{2}}{\partial x^{2}}+\frac{\rho J}{\kappa G A} \frac{\partial^{2}}{\partial t^{2}}\right] P \delta(x-v t) .
\end{aligned}
$$


The motion equation of Eq. (52) is complex and, if only the external motion resistances depending on damping coefficient $c\left[\mathrm{Ns} / \mathrm{m}^{2}\right]$ are considered and retardation time $\tau$ is neglected, assumes the form

$$
\begin{aligned}
& \overline{E J}\left(1+\frac{G_{s}}{\kappa G A}\right) \frac{\partial^{4} w}{\partial x^{4}} \\
& -\rho\left(J+\frac{\overline{E J}}{\kappa G}+\frac{G_{s} J}{\kappa G A}+m_{s} \frac{\overline{E J}}{\kappa G A}\right) \frac{\partial^{4} w}{\partial x^{2} \partial t^{2}} \\
& +\frac{\rho J}{\kappa G}\left(\rho+\frac{m_{s}}{A}\right) \frac{\partial^{4} w}{\partial t^{4}}+\frac{\rho J c}{\kappa G A} \frac{\partial^{3} w}{\partial t^{3}} \\
& -\frac{\overline{E J} c}{\kappa G A} \frac{\partial^{3} w}{\partial x^{2} \partial t}-\left(G_{s}+\frac{\overline{E J} k_{s}}{\kappa G A}\right) \frac{\partial^{2} w}{\partial x^{2}} \\
& +\left(\rho A+\frac{\rho J k_{s}}{\kappa G A}+m_{s}\right) \frac{\partial^{2} w}{\partial t^{2}}+c \frac{\partial w}{\partial t}+k_{s} w \\
& =\left(1-\frac{\overline{E J}}{\kappa G A} \frac{\partial^{2}}{\partial x^{2}}+\frac{\rho J}{\kappa G A} \frac{\partial^{2}}{\partial t^{2}}\right) P \delta(x-v t) .
\end{aligned}
$$

Damping coefficient $c$ can be treated as a substitute damping characteristic that represents the internal damping in the beam and the external damping in the foundation alike.

\section{Numerical examples}

Based on the equations presented in previous sections and their solutions, the beam vibrations caused by passing a force moving at a constant velocity have been analyzed. Impact has been examined of the foundation properties (elastic settlement, shear, and inertia factors), as well as of inhomogeneity (transversal variability of Young's modulus), on the beam deflection. Critical velocities have been determined and dynamic coefficients relating to beam deflections analyzed. Two vibrations cases were considered: when a moving force is applied to the beam (forced vibration), and after the moving force has been left the beam (free vibration). The graphs are shown in dimensionless coordinates. The beam's vertical displacement has been related to the static deflection of the center of Timoshenko beam span $w_{s t}^{T}$, whereas the moving force position has been related to the beam length $l$. For the results' analysis and graphic rendering Mathematica was used.

\subsection{Beam deflection analysis}

Timoshenko beam resting on inertial Vlasov foundation, which can be used to model a road pavement fragment, is analyzed. Therefore, we will consider a structure of cement concrete on a lean concrete foundation. Let's assume that there is no slip between the beam layers. The following beam material data have been assumed for the calculation: the upper concrete layer's elastic modulus $E_{1}=36.0 \cdot 10^{9} \mathrm{~Pa}$, foundation's elastic modulus $E_{2}=20.8 \cdot 10^{9} \mathrm{~Pa}$, Poisson's ratio of both concrete layers $v=0.16$, beam material density $\rho=2400 \mathrm{~kg} / \mathrm{m}^{3}$. Beam span $l=3.6 \mathrm{~m}$, rectangular cross-section's shape factor $\kappa=5 / 6$. The beam layers' thicknesses are assumed equal to $h_{1}=0.27 \mathrm{~m}$ and $h_{2}=0.18 \mathrm{~m}$, and their width to $b=0.30 \mathrm{~m}$. Since the structure consists of two layers with different elastic moduli, an equivalent Young's modulus (equivalent beam stiffness $\bar{E} \bar{J}$ ) is adopted for the calculation. It is also assumed that the $H=1.50 \mathrm{~m}$ thick soil layer under the foundation is characterized by Poisson's ratio $v_{s}=0.30$ and Young's modulus $E_{s}=30 \cdot 10^{6} \mathrm{~Pa}, E_{s}=50 \cdot 10^{6} \mathrm{~Pa}$ or $E_{s}=100 \cdot 10^{6} \mathrm{~Pa}$, which in the first case, for example, corresponds to the ground parameters in Vlasov model $k_{s}=8.08 \mathrm{MPa}, G_{s}=0.86 \mathrm{MN}$ and $m_{s}=397.55 \mathrm{~kg} / \mathrm{m}$.

The foundation's impact on the beam midpoint vibration is shown in the charts in Fig. 2. Charts of the tracking deflection under a moving force, i.e. at $x=v t$, in case of $v=60 \mathrm{~m} / \mathrm{s}$, are shown in Fig. 3. Whereas the beam midpoint vibrations depending on the moving load velocity is shown in Fig. 4. The beam deflections in Figs. 2-4 refer to the static deflection of the midpoint of a beam loaded in its span center, without the foundation's consideration.

\subsection{Damping effect}

The external damping effect on vibration of Timoshenko beam resting on Vlasov layer and subjected to a moving force will be analyzed by solving the beam motion equation Eq. (53), assuming the simple support conditions. The solutions for vibrations forced by a force movement and free vibrations have been obtained using Mathematica.

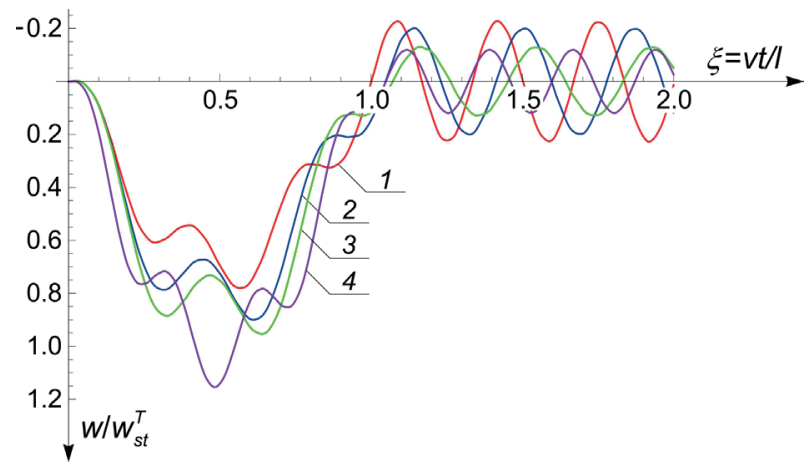

Fig. 2 Foundation parameters' impact on beam midpoint deflection $v=60 \mathrm{~m} / \mathrm{s}: 1-$ beam on Vlasov foundation with $E_{s}=100 \cdot 10^{6} \mathrm{~Pa}$; $2-E_{s}=50 \cdot 10^{6} \mathrm{~Pa} ; 3-E_{s}=30 \cdot 10^{6} \mathrm{~Pa} ; 4-$ with foundation impact neglected 


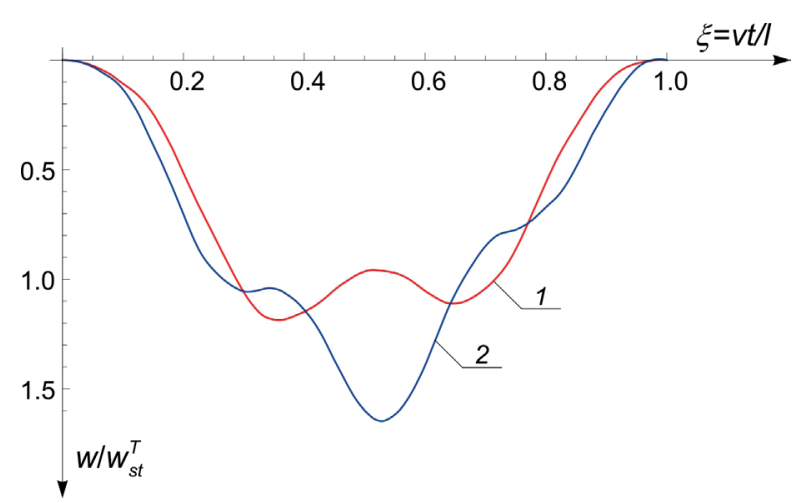

Fig. 3 Deflection $w(x=v t, t)$ under concentrated force; 1 - beam on Vlasov foundation with $E_{s}=30 \cdot 10^{6} \mathrm{~Pa} ; 2$ - with foundation impact neglected, moving force velocity in both cases $v=60 \mathrm{~m} / \mathrm{s}$

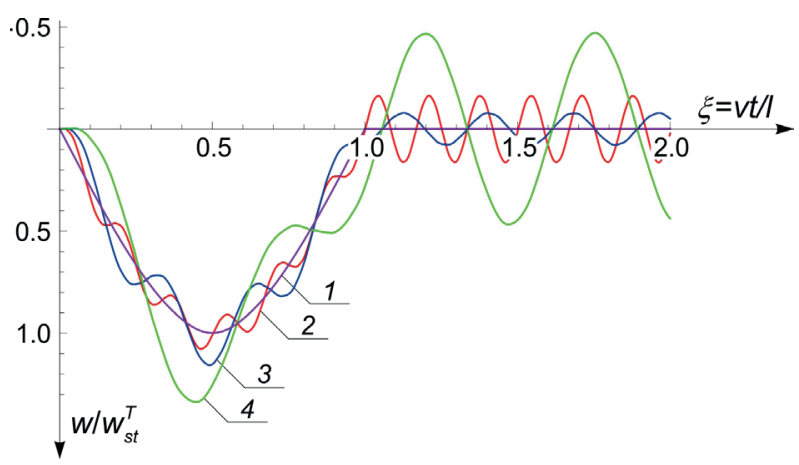

Fig. 4 Dynamic deflection of the midpoint of Timoshenko beam on Vlasov foundation with $E_{s}=100 \cdot 10^{6} \mathrm{~Pa} ; 1-$ static influence line; 2 - moving force velocity $v=30 \mathrm{~m} / \mathrm{s} ; 3-v=50 \mathrm{~m} / \mathrm{s} ; 4-v=100 \mathrm{~m} / \mathrm{s}$

The calculations were made using the same numerical data of a beam on a foundation as in the previous calculation examples in this section. In addition, damping number $\zeta=\left(c / 2 \bar{m} \omega_{1}\right) \cdot 100 \%$ was introduced into the calculation. The results are illustrated in Fig. 5 and Fig. 6 by the beam midpoint's damped vibration charts. Quantity $w_{s t}^{T}$ here is the static deflection of the midpoint of a simply supported beam resting on a foundation, and loaded in the middle of the span with a force.

\subsection{Effect of moving force velocity}

In the relevant literature, there are several dynamic factor definitions, e.g. in [22]. As shown in these studies, dynamic factors differ in the cases of dynamic beam deflections, dynamic bending moments, and in the case of dynamic shear forces.

In this study the effect of the moving force's velocity on the dynamic deflections of simply supported Timoshenko beam resting on Vlasov layer is examined. The dynamic factors are defined by formulas

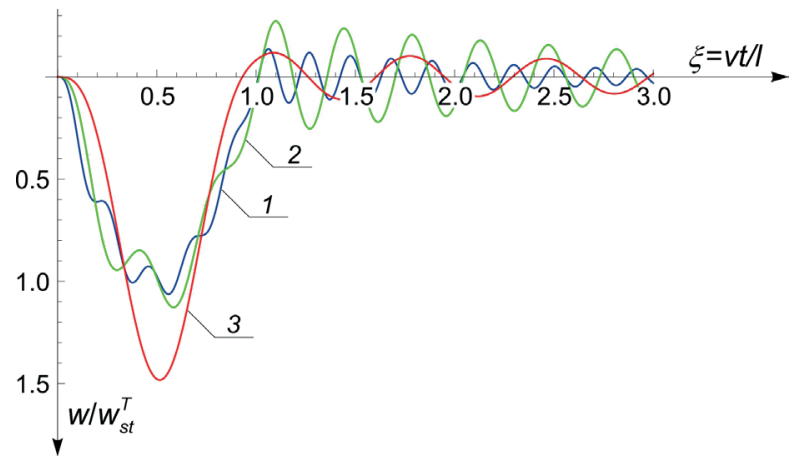

Fig. 5 Velocity influence on damped vibration $(\zeta=5 \%)$ of the midpoint of Timoshenko beam resting on Vlasov layer $\left(E_{s}=30 \cdot 10^{6} \mathrm{~Pa}\right)$;

$1-$ moving force velocity $v=30 \mathrm{~m} / \mathrm{s} ; 2-v=50 \mathrm{~m} / \mathrm{s} ; 3-v=100 \mathrm{~m} / \mathrm{s}$

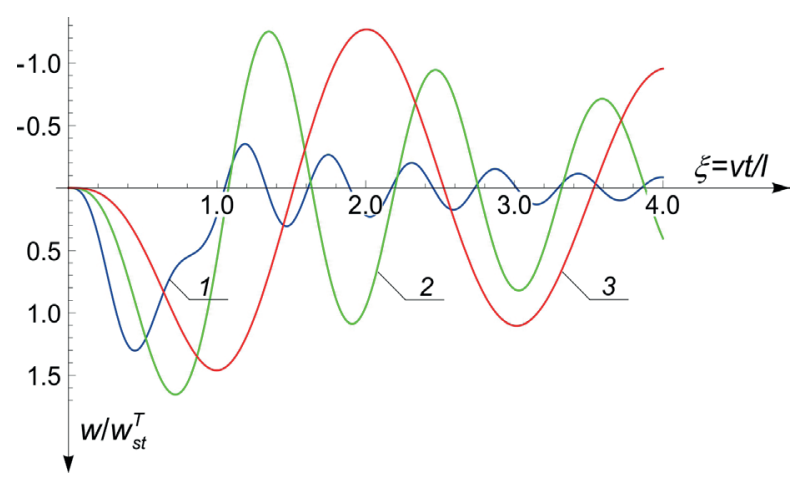

Fig. 6 Velocity influence on damped vibration $(\zeta=10 \%)$ of the midpoint of Timoshenko beam resting on Vlasov layer, $E_{s}=100 \cdot 10^{6} \mathrm{~Pa}$;

1 - moving force velocity $v=0.25 v_{c r} ; 2-v=0.50 v_{c r} ; 3-v=0.90 v_{c r}$

$n_{d}^{*}=\frac{\max [w(l / 2, t)]}{w_{s t}^{*}(l / 2)}$

$n_{d}=\frac{\max [w(l / 2, t)]}{w_{s t}(l / 2)}$,

for

$t \in\left(0, \frac{l}{v}\right)$

Where $w_{s t}^{*}(l / 2)$ means the static deflection of the midpoint of a simply supported beam, without consideration of the foundation, whereas $w_{s t}(l / 2)$ is the static deflection of the midpoint of a beam on a foundation.

The relationship between moving force velocity $v$ and foundation elastic modulus $E$, and dynamic factor $n_{d}^{*}$ is presented in the three-dimensional space in Fig. 7. The charts with coordinates $E_{s}, n_{d}^{*}$, in three cases: static $(v=0 \mathrm{~m} / \mathrm{s})$ and in two dynamic cases $(v=50 \mathrm{~m} / \mathrm{s}$ and $v=100 \mathrm{~m} / \mathrm{s}$ ), are shown in Fig. 8. Similar charts, but with Vlasov layer considered in the beam's static deflection determination, are shown in Fig. 9. and Fig. 10. 
In addition, Fig. 11 shows a chart of dynamic factor $n_{d}$ when changing the moving force velocity from zero to $0.5 v_{c r}=214.5 \mathrm{~m} / \mathrm{s}$ (soil foundation with elastic modulus $\left.E_{s}=30 \mathrm{MPa}\right)$.

The resulting solutions' convergence is confirmed, inter alia, by phase portraits in coordinates $w, \dot{w}$. One of these charts is shown in Fig. 12.

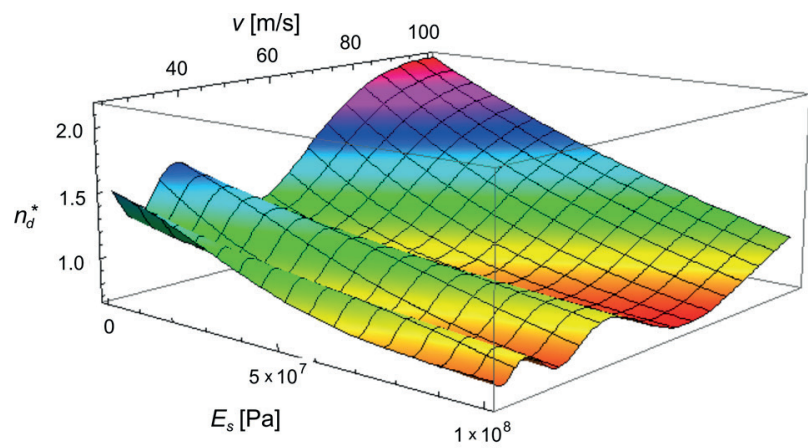

Fig. 7 Effect of moving force velocity and foundation Young's modulus on deflection of the midpoint of simply supported beam, dynamic factor $n_{d}{ }^{*}$ depending on static beam deflection with foundation influence neglected

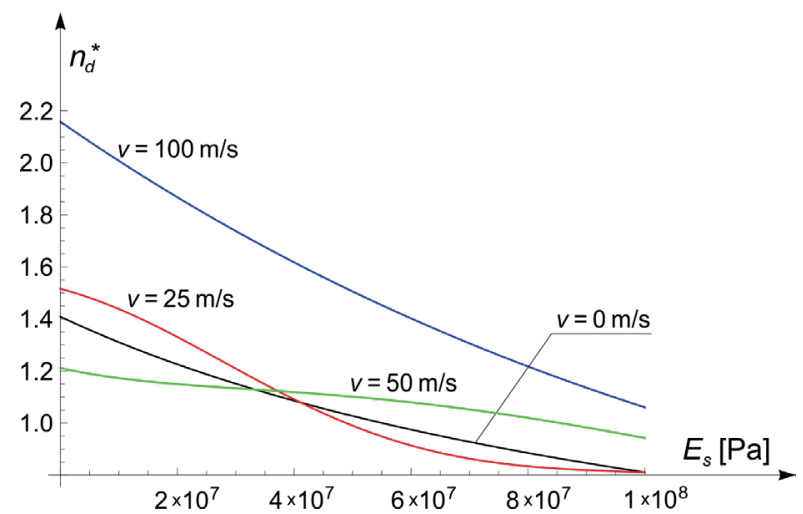

Fig. 8 Effect of moving force velocity and foundation Young's modulus on deflection of the midpoint of simply supported beam, dynamic factor $n_{d}$ " depending on static beam deflection with foundation influence neglected

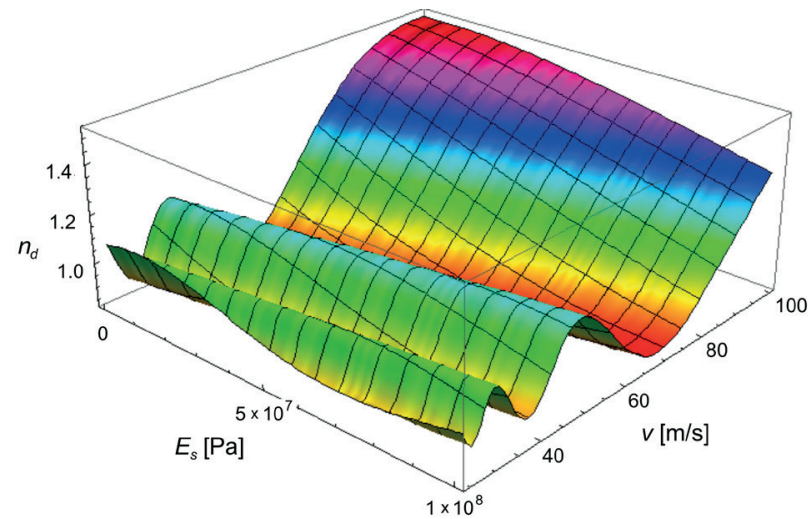

Fig. 9 Effect of moving force velocity and foundation Young's modulus on deflection of the midpoint of simply supported beam, dynamic factor $n_{d}$ depending on the static deflection of beam on Vlasov foundation

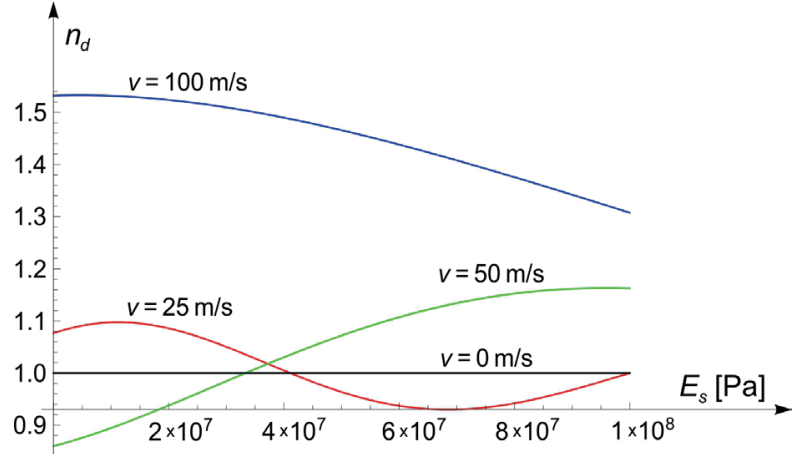

Fig. 10 Effect of moving force velocity and foundation elastic modulus on deflection of the midpoint of simply supported beam, dynamic factor $n_{d}$ depending on the static deflection of beam on Vlasov foundation

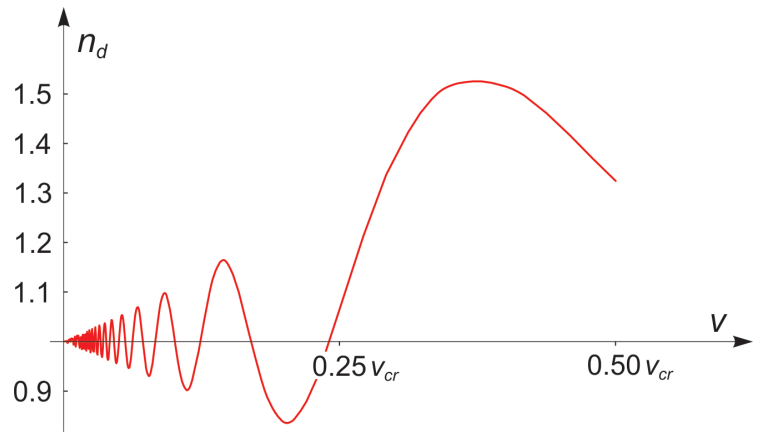

Fig. 11 Variation of dynamic factor $n_{d}$ when changing moving force velocity from zero to $0.5 v_{c r}$, soil foundation's elastic modulus $E_{s}=30 \mathrm{MPa}$

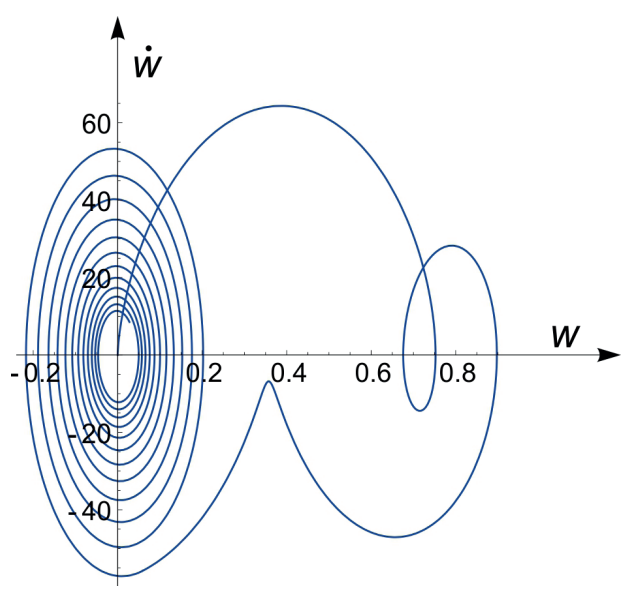

Fig. 12 Phase portrait in coordinates $w$ and $\dot{w}$ in case of beam on Vlasov foundation with parameters $k=8.08 \mathrm{MPa}, G_{s}=0.86 \mathrm{MN}$, $m_{s}=397.55 \mathrm{~kg} / \mathrm{m}\left(E_{s}=30 \mathrm{MPa}\right)$

\section{Conclusions}

Based on this analysis it can be concluded that the deflections from the vibration at circular frequency $\omega_{2 n}$ are small compared to the purely forced vibration at frequency $\alpha_{n} v$, as well as the associated free vibration at frequency $\omega_{1 n}$. Thus, ignoring components containing $\omega_{2 n}$ in practical applications it is justified. 
The results indicate that appropriate selection of the foundation's parameters allows for the beam deflection's significant reduction, while the impact of the shear coefficient in the foundation on the reduction is more pronounced than the impact of other factors.

Both the moving force velocity and the foundation also influence the dynamic factors, which are different in the cases of dynamic beam deflections, dynamic bending moments, and dynamic transverse forces. The dynamic factors strongly depend on the moving force velocity and are variable, increasing and decreasing alternately as the velocity's function.

\section{References}

[1] Timoshenko, S. P. "On the correction for shear of the differential equation for transverse vibrations of prismatic bars", Philosophical Magazine, 41(245), pp. 744-746, 1921.

https://doi.org/10.1080/14786442108636264

[2] Timoshenko, S. P. "On the transverse vibrations of bars of uniform cross-section", Philosophical Magazine, 43(253), pp. 125-131, 1922. https://doi.org/10.1080/14786442208633855

[3] Laura, P. A. A., Maurizi, M. J., Rossi, R. E. "A survey of studies dealing with Timoshenko beams", The Shock and Vibration Digest, 22(11), pp. 3-10, 1990. https://doi.org/10.1177/058310249002201102

[4] Mindlin, R. D. "An Introduction to the Mathematical Theory of Vibrations of Elastic Plates", 1st ed., World Scientific, Hackensack, NJ, USA, 2006.

https://doi.org/10.1142/6309

[5] Szcześniak, W. "Drgania swobodne lepkosprężystej belki Timoshenki i tarczy" (Free vibrations of viscoelastic Timoshenko beam and shield), Engineering Transactions, 22(4), pp. 669-687, 1974. (in Polish)

[6] Abbas, B. A. H., Thomas, J. "The second frequency spectrum of timoshenko beams", Journal of Sound and Vibration, 51(1), pp. 123-137, 1977.

https://doi.org/10.1016/S0022-460X(77)80118-1

[7] O'Reilly, O. M., Turcotte, J. S. "Another mode of vibration in a Timoshenko beam", Journal of Sound and Vibration, 198(4), pp. 517-521, 1996

https://doi.org/10.1006/jsvi.1996.0585

[8] Han, S. M., Benaroya, H., Wei, T. "Dynamics of transversely vibrating beams using four engineering theories", Journal of Sound and Vibration, 225(5), pp. 935-988, 1999. https://doi.org/10.1006/jsvi.1999.2257

[9] Śniady, P. "Dynamic Response of a Timoshenko Beam to a Moving Force", Journal of Applied Mechanics, 75(2), 2008. https://doi.org/10.1115/1.2775500

[10] Huang, C.-C. "Travelling Loads on a Viscoelastic Timoshenko Beam", Journal of Applied Mechanics, 44(1), pp. 183-184, 1977. https://doi.org/10.1115/1.3423995
[11] Wang, T. M., Gagnon, L. W. "Vibration of continuous Timoshenko beams on Winkler-Pasternak foundations", Journal of Sound and Vibration, 58(2), pp. 211-220, 1978. https://doi.org/10.1016/0022-460X(78)90501-1

[12] Mackertich, S. "Moving load on a Timoshenko beam", Journal of the Acoustical Society of America, 88(2), pp. 1175-1178, 1990. https://doi.org/10.1121/1.399814

[13] Felszeghy, S. F. "The Timoshenko Beam on an Elastic Foundation and Subject to a Moving Step Load, Part 1: Steady-State Response", Journal of Vibration and Acoustics, 118(3), pp. 277-284, 1996. https://doi.org/10.1115/1.2888178

[14] Felszeghy, S. F. "The Timoshenko Beam on an Elastic Foundation and Subject to a Moving Step Load, Part 2: Transient Response", Journal of Vibration and Acoustics, 118(3), pp. 285-291, 1996. https://doi.org/10.1115/1.2888179

[15] Lee, H. P. "Dynamic response of a Timoshenko beam on a winkler foundation subjected to a moving mass", Applied Acoustic, 55(3), pp. 203-215, 1998. https://doi.org/10.1016/S0003-682X(97)00097-2

[16] Chen, Y.-H., Huang, Y.-H., Shih, C.-T. "Response of an infinite Timoshenko beam on a viscoelastic foundation to a harmonic moving load", Journal of Sound and Vibration, 241(5), pp. 809-824, 2001. https://doi.org/10.1006/jsvi.2000.3333

[17] Bystrzycki, J. "Równania ruchu belek sprężystych. Belka Timoshenki" (The equations of motion of elastic beams. Timoshenko beam), IFTR Reports, Warsaw, Poland, 1977. (in Polish)

[18] Mindlin, R. D., Deresiewicz, H. "Timoshenko's shear coefficient for flexural vibrations of beams", In: Proceedings 2nd U.S. National Congress of Applied Mechanics, Ann Arbor, MI, USA, 1954, pp. 175-178.

[19] Vlasov, V. Z., Leontev, N. N. "Балки, плиты и оболочки на упругом основании" (Beams, plates and shells on elastic foundations), GIFML, Moscow, Russia, 1960. (in Russian)

[20] Nowiński, J., Turski, S. "Studium nad stanami naprężenia w ciałach sprężystych niejednorodnych" (Study on stress states in nonhomogeneous elastic bodies), Archives of Mechanics, 5(3), 1953. (in Polish)

[21] Szcześniak, W. "Warunki początkowe w zagadnieniu dynamicznym belki Timoshenki" (Initial conditions in the dynamic problem of Timoshenko's beam), Warsaw University of Technology Research Papers, Construction, 108, pp. 99-143, 1989. (in Polish)

[22] Yang, Y. B., Yau, J. D., Wu, Y. S. "Vehicle-Bridge Interaction Dynamics", World Scientific, Singapore, 2004. https://doi.org/10.1142/5541 